\title{
Exponentiation and Euler measure
}

\author{
James Propp \\ University of Wisconsin \\ April 29, 2001 \\ Revised May 29, 2002 \\ dedicated to the memory of Gian-Carlo Rota
}

\begin{abstract}
Two of the pillars of combinatorics are the notion of choosing an arbitrary subset of a set with $n$ elements (which can be done in $2^{n}$ ways), and the notion of choosing a $k$-element subset of a set with $n$ elements (which can be done in $\left(\begin{array}{l}n \\ k\end{array}\right)$ ways). In this article I sketch the beginnings of a theory that would import these notions into the category of hedral sets in the sense of Morelli and the category of polyhedral sets in the sense of Schanuel. Both of these theories can be viewed as extensions of the theory of finite sets and mappings between finite sets, with the concept of cardinality being replaced by the more general notion of Euler measure (sometimes called combinatorial Euler characteristic). I prove a "functoriality" theorem (Theorem 1) for subset-selection in the context of polyhedral sets, which provides quasi-combinatorial interpretations of assertions such as $2^{-1}=\frac{1}{2}$ and $\left(\begin{array}{c}1 / 2 \\ 2\end{array}\right)=-\frac{1}{8}$. Furthermore, the operation of forming a power set can be viewed as a special case of the operation of forming the set of all mappings from one set to another; I conclude the article with a polyhedral analogue of the set of all mappings between two finite sets, and a restrictive but suggestive result (Theorem 2) that offers a hint of what a general theory of exponentiation in the polyhedral category might look like. (Other glimpses into the theory may be found in [Pro].)
\end{abstract}

\section{Introduction}

In the work of Hadwiger [Ha1] [Ha2], Lenz [Len], McMullen [McM], Rota [KR], Chen [Ch1] [Ch2], and others, the Euler characteristic of polytopes in $\mathbf{R}^{n}$ is extended to a valuation $\chi$ (the Euler measure) on the algebra of sets generated by these polytopes. Included in this algebra of sets (called 
"hedral sets" by Morelli [Mor] and "polyhedral sets" by Schanuel [Sch]) are the finite subsets of $\mathbf{R}^{n}$, for which the notion of Euler measure and the notion of cardinality coincide. This suggests the viewpoint (forcefully advocated by Schanuel) that we view polyhedral sets and their $\mathbf{Z}$-valued Euler measures as a generalization of finite sets and their $\mathbf{N}$-valued cardinalities. Indeed, several of the key pillars on which combinatorics rests - concepts of union, Cartesian product and selection of a subset of fixed size - extend to the polyhedral setting straightforwardly, and this extension provides a natural interpretation for assertions like $\left(\begin{array}{c}-2 \\ 3\end{array}\right)=-4$ (see section 2).

What else constitutes the foundation of combinatorics? Many combinatorialists would point to the notion of the set of all maps (or injections, or surjections, or bijections) from one finite set to another; see for instance the "twelve-fold way" of Rota (so dubbed by Spencer) as described in Stanley's book [Sta]. As a special case, the set of all maps from a finite set to the set $\{0,1\}$ corresponds naturally with the set of all subsets of the given set.

I will show that a polyhedral analogue of this foundation can be built, using Morelli's notion of hedral maps (piecewise-constant maps with finite range whose level sets are polyhedral sets) and Schanuel's more inclusive notion of polyhedral maps (maps whose graphs are polyhedral sets). Since in neither case is the set of all such maps from $A$ to $B$ itself a polyhedral set (except when $A$ and $B$ are finite or $A$ or $B$ is empty), we cannot simply take Euler measure in the standard way; but, at least in the case where the polyhedral set $A$ is a subset of $\mathbf{R}$, we can divide this large set of maps into finitedimensional strata by limiting the number of points of discontinuity/nondifferentiability and use this stratification to define a power series in $t$ whose value at $t=1$ "should" be the Euler measure of the set of maps. In many cases the series possesses an analytic continuation with a well-defined value at $t=1$, which I call the regularized Euler measure.

For instance, let $A=(0,1)$ and $B=\{0,1\}$, with $\chi(A)=-1$ and $\chi(B)=2$; then we will see in section 5 that $B^{A}$, defined as the set of all polyhedral maps from $A$ to $B$, has regularized Euler measure $\frac{1}{2}$, and the set of all two-element subsets of $B^{A}$ has regularized Euler measure $-\frac{1}{8}$. The preceding assertions correspond, in some way not yet formalized, to the algebraic assertions that $\frac{1}{2}=2^{-1}=\chi(B)^{\chi(A)}$ and $-\frac{1}{8}=\left(\begin{array}{c}1 / 2 \\ 2\end{array}\right)$ (under the interpretation of $\left(\begin{array}{l}x \\ k\end{array}\right)$ as a polynomial of degree $k$ in $\left.x \in \mathbf{R}\right)$.

A different theory of exponentiation of polyhedral sets arises from focus- 
ing on power sets, and making the restriction that one looks at only the finite subsets of a given polyhedral set. Since this theory is simpler and has been developed further, it is discussed first, in sections 3 and 4 . Then, in section 5, I treat the set of maps from one polyhedral set to another, under both Morelli's condition of hedrality and Schanuel's weaker condition of polyhedrality.

I should warn the reader not to expect too much; the two theorems proved in this article are really more like examples than like theorems - and what is more, I do not give a clear statement of what sorts of definitions and theorems these examples are special cases of. It is my hope that at least some readers will see that there are issues here that are worthy of being pursued, and that there is some "spiritual" satisfaction in the prospect of a theory that might be a model of the rational numbers in the same way that ordinary combinatorics is a model of the non-negative integers.

I thank Scott Axelrod, John Baez, Beifang Chen, Timothy Chow, Ezra Getzler, Greg Kuperberg, Michael Larsen, Ayelet Lindenstrauss, Haynes Miller, Lauren Rose, and Dylan Thurston for helpful conversations. I also thank Joseph Kung for soliciting this article and putting this special issue together, and the anonymous referee for numerous helpful suggestions.

I acknowledge a special debt to Gian-Carlo Rota, who persistently encouraged the investigations described here, despite their inconclusive status both in 1996 (when I described my work to him) and now (after years of neglect). I also want to acknowledge the formative role he played in my career as the author of the beautiful book Finite Operator Calculus, which was part of what inspired my earliest explorations of combinatorics. His sagacity and warmth are missed by me and many others.

\section{Background}

Following Schanuel, we define a polyhedral set in $\mathbf{R}^{n}$ as one that can be defined by a Boolean predicate whose primitive terms are linear equations and inequalities in $n$ variables. Equivalently, the algebra of polyhedral sets is the algebra generated by the closed half-planes in $\mathbf{R}^{n}$ under intersection, union and complementation. Note that polyhedral sets can be unbounded; for instance, $\{(x, y):|x|+|y| \leq 1\} \cup\{(x, 0): x>2\} \cup\{(5,5)\}$ is a polyhedral subset of $\mathbf{R}^{2}$.

There exists a unique function $\chi(\cdot)$ from the polyhedral algebra to the 
integers such that

$$
\chi(A \cup B)=\chi(A)+\chi(B)-\chi(A \cap B)
$$

for all polyhedral sets $A, B$ and such that

$$
\chi(A)=(-1)^{k}
$$

if the polyhedral set $A$ is homeomorphic to $\mathbf{R}^{k}$ or equivalently is homeomorphic to the interior of a $k$-dimensional disk (see e.g. [Ch2]). For instance, the polyhedral set described at the end of the preceding paragraph has $\chi$ value $1-1+1=1$. The function $\chi$, hereafter called simply Euler measure, is invariant under homeomorphism, and in the case of compact polyhedral sets, it coincides with the alternating sum of the Betti numbers. However, unlike the notion of Euler characteristic favored by topologists, it is not a homotopy invariant (e.g., $\chi$ of an open interval in $\mathbf{R}$ is -1 whereas $\chi$ of a closed interval is +1 ). Property (1) extends naturally to an inclusion-exclusion principle:

$$
\begin{aligned}
& \chi\left(A_{1} \cup A_{2} \cup \ldots \cup A_{m}\right)= \\
& \sum_{1 \leq i \leq m} \chi\left(A_{i}\right)-\sum_{1 \leq i<j \leq m} \chi\left(A_{i} \cap A_{j}\right)+\ldots \pm \chi\left(A_{1} \cap A_{2} \cap \ldots \cap A_{m}\right) .
\end{aligned}
$$

For polyhedral sets $A \subset \mathbf{R}^{m}$ and $B \subset \mathbf{R}^{n}$, one obtains a Cartesian product $A \times B$ in $\mathbf{R}^{m+n}$ in the obvious way, and it can easily be shown that $\chi(A \times B)=\chi(A) \chi(B)$. Less trivially, given a polyhedral set $A \subset \mathbf{R}^{n}$ and an integer $k \geq 0$, there is a way of defining a polyhedral set $\left(\begin{array}{l}A \\ k\end{array}\right) \subset\left(\mathbf{R}^{n}\right)^{k}$ that contains exactly one point in each free orbit in the $k$-fold Cartesian product $A^{k}$ under the permutation action of the symmetric group $S_{k}$ (where the free orbits are those of cardinality $k$ !, i.e., the orbits of $n$-tuples of distinct real numbers); this construction is well-defined up to polyhedral isomorphism (defined below) and has the property that $\chi\left(\left(\begin{array}{l}A \\ k\end{array}\right)\right)=\left(\begin{array}{c}\chi(A) \\ k\end{array}\right)$. Since the operation $A \mapsto\left(\begin{array}{l}A \\ k\end{array}\right)$ (mapping Schanuel's category of polyhedral sets to itself) seems to be new, it is necessary to amplify on the preceding sentences in the following paragraphs.

Following Schanuel, we define a polyhedral map from a polyhedral set $A \subset \mathbf{R}^{m}$ to a polyhedral set $B \subset \mathbf{R}^{n}$ as a map $A \rightarrow B$ whose graph in $\mathbf{R}^{m+n}$ is itself a polyhedral set; that is, a polyhedral map is a piecewise-affine 
map that need not be continuous. A polyhedral isomorphism is a bijective polyhedral map, such as the map

$$
f(x)= \begin{cases}x-1 & \text { if }-1<x<0 \\ x+5 & \text { if } x=0 \\ 2 x+1 & \text { if } 0<x<1\end{cases}
$$

between the interval $(-1,1) \subset \mathbf{R}$ and the set $(-2,-1) \cup(1,3) \cup\{5\} \subset \mathbf{R}$. It can be shown that two polyhedrally isomorphic polyhedral sets have the same Euler measure. On the other hand, following Morelli, we define a hedral map from a polyhedral set $A \subset \mathbf{R}^{m}$ to a polyhedral set $B \subset \mathbf{R}^{n}$ to be a function $f: A \rightarrow B$ with finite range whose level-sets are all polyhedral.

In both cases, we identify a function $f: \mathbf{R}^{m} \rightarrow \mathbf{R}^{n}$ with its graph in $\mathbf{R}^{m+n}$.

Note that if $A$ is finite, the hedral and polyhedral maps from $A$ to $B$ are just the set-theoretic maps, so that, in the case where both $A$ and $B$ are finite, the set of hedral or polyhedral maps from $A$ to $B$ has cardinality $|B|^{|A|}$ (or, in other terms, Euler measure $\chi(B)^{\chi(A)}$ ). If $A$ is infinite, then most set-theoretic maps from $A$ to $B$ are neither hedral nor polyhedral.

Also note that when $B$ is finite, the the set of hedral maps from $A$ to $B$ coincides with the set of polyhedral maps from $A$ to $B$; when $B$ is infinite, every hedral map is polyhedral but not conversely.

We now define a polyhedral ordering on a polyhedral set $A \subset \mathbf{R}^{n}$ as a (total) linear ordering whose graph in $A \times A$ is polyhedral; an example of such an ordering is the one induced by lexicographic ordering of $n$-tuples in $\mathbf{R}^{n}$. Henceforth, we restrict attention to lexicographic ordering. We form $\left(\begin{array}{l}A \\ k\end{array}\right) \subset A^{k}$ by selecting, from each free orbit in the $k$-fold Cartesian product $A^{k}$ under the permutation action of the symmetric group $S_{k}$, the one $k$-tuple that is lexicographically least in the induced ordering on $A^{k}$. That is, $\left(\begin{array}{l}A \\ k\end{array}\right)$ consists of all $k$-tuples $\left(v_{1}, v_{2}, \ldots, v_{k}\right) \in A^{k}$ with $v_{1}<v_{2}<\ldots<v_{k}$ in the lexicographic sense. (Note that any polyhedral set that contains exactly one point in each free orbit in $A^{k}$ under the action of $S_{k}$ is canonically equivalent to the one defined above, via a polyhedral isomorphism.)

Take for instance $A=(0,1) \cup(2,3) \subset \mathbf{R}$, with $\chi(A)=-2$. Then we find that $\left(\begin{array}{l}A \\ 3\end{array}\right)$ is a union of four sets: $\{(x, y, z): 0<x<y<z<1\}$, $\{(x, y, z): 0<x<y<1,2<z<3\},\{(x, y, z): 0<x<1,2<y<z<3\}$ and $\{(x, y, z): 2<x<y<z<3\}$. Each of these is homeomorphic to the 
interior of a 3-ball, so $\chi\left(\left(\begin{array}{l}A \\ 3\end{array}\right)\right)=(-1)^{3}+(-1)^{3}+(-1)^{3}+(-1)^{3}=-4$. Note that this is also the value of $\left(\begin{array}{c}\chi(A) \\ 3\end{array}\right)$ where for each fixed $k \geq 0$ and all $x \in \mathbf{R}$ we define $\left(\begin{array}{l}x \\ k\end{array}\right)$ as $x(x-1) \cdots(x-k+1) / k !$.

To show that $\chi\left(\left(\begin{array}{l}A \\ k\end{array}\right)\right)=\left(\begin{array}{c}\chi(A) \\ k\end{array}\right)$ for all polyhedral sets $A$ and all integers $k \geq 0$, it suffices to show that the polyhedral set $\left\{\left(v_{1}, v_{2}, \ldots, v_{k}\right) \in A^{k}: v_{i} \neq\right.$ $v_{j}$ for all $\left.i \neq j\right\}$ has Euler measure $\chi(A)(\chi(A)-1) \cdots(\chi(A)-k+1)$, since this larger polyhedral set is the disjoint union of $k$ ! polyhedrally-isomorphic copies of $\left(\begin{array}{l}A \\ k\end{array}\right)$. One way to prove this is by iterated application of a Fubini theorem (Theorem 3 in Morelli): If a polyhedral set $A \subset B \times C$ has the property that every $C$-fiber has Euler measure $m$, then $A$ has Euler measure $\chi(B) \cdot m$. For example, in the case $k=2$, it is clear that $\left\{\left(v_{1}, v_{2}\right) \in A^{2}: v_{1} \neq v_{2}\right\}$ projects to its first factor with fibers of Euler measure $\chi(A)-1$, so by the Fubini principle its Euler measure must be $\chi(A)(\chi(A)-1)$; the same approach works when applied iteratively in the case of larger values of $k$. This proof has the virtue of being entirely parallel to the standard way of counting ordered $k$-element subsets of a finite set, and it makes it clear where the factors $\chi(A)-i$ come from. However, as an intimation of what is to come, it is more helpful to prove the result by poset-theoretic inclusion-exclusion, as we now do. (See Stanley's book [Sta] for background on partially ordered sets.)

The partition lattice $\Pi_{k}$ is the set of unordered partitions of a $k$-element set, ordered so that its top element is the 1-block partition $\hat{1}$ and its bottom element is the $k$-block partition $\hat{0}$. It is easy to see that for any finite set $S$, the product set $S^{n}=\left\{\left(s_{1}, s_{2}, \ldots, s_{n}\right)\right\}$ splits up into $n$ disjoint subsets, where the $k$ th subset (with $k$ between 1 and $n$ inclusive) consists of those $n$-tuples that contain exactly $k$ distinct elements of $S$ and therefore has cardinality $|S|^{k}$. Ordinary inclusion-exclusion applied to the lattice $\Pi_{k}$ lets us turn this around and conclude that the number of ordered $k$-element subsets of $S$ is

$$
\sum_{\pi \in \Pi_{k}} \mu(\hat{0}, \pi)|S|^{N(\pi)}
$$

where $\mu(\cdot, \cdot)$ is the Möbius function of $\Pi_{k}$ and $N(\pi)$ is the number of blocks in the partition $\pi$. (See exercise 44 of [Sta].) Proceeding analogously in the case of polyhedral sets, we see that for any polyhedral set $A$, the product polyhedron $A^{n}=\left\{\left(v_{1}, v_{2}, \ldots, v_{n}\right)\right\}$ splits up into $n$ disjoint polyhedral sets, where the $k$ th polyhedral set (with $k$ between 1 and $n$ inclusive) consists of those $n$-tuples that contain exactly $k$ distinct elements of $A$ and therefore has 
Euler measure $\chi(A)^{k}$. As before, inclusion-exclusion applied to the lattice $\Pi_{k}$ tells us that the Euler measure of $\left(\begin{array}{l}A \\ k\end{array}\right)$ is

$$
\sum_{\pi \in \Pi_{k}} \mu(\hat{0}, \pi) \chi(A)^{N(\pi)}
$$

Since the two polynomials are the same (aside from the fact that one has $\chi(A)$ where the other has $|S|)$, and since we know that the former is equal to $|S|(|S|-1) \cdots(|S|-k+1)$, the desired result follows.

(The operations $\lambda^{k}: A \mapsto\left(\begin{array}{l}A \\ k\end{array}\right)$ are akin to the $\lambda$-ring structures that Morelli defines on the abelian group of hedral functions, in Theorem 13 of his article and the Remark that follows it.)

As an aside, it is interesting to note that although finding a polyhedral analogue of $n \mapsto 2^{n}$ seem to require us to go beyond the polyhedral category and into an extension involving infinite-dimensional objects, finding a polyhedral analogue of the Fibonacci numbers does not. Given a polyhedral set $P \subset \mathbf{R}$ of Euler measure $n$ (positive, negative, or zero), the set of all finite subsets $S \subset P$ such that $\chi\left((P \backslash S) \cap\left(t, t^{\prime}\right)\right)$ is even for all $t, t^{\prime} \in S \cup\{-\infty,+\infty\}$ has Euler measure $\left(\phi^{n+1}-(-1 / \phi)^{n+1}\right) / \sqrt{5}$ (with $\left.\phi=(1+\sqrt{5}) / 2\right)$.

\section{The Small Power Set}

To motivate our definition of the Euler series, we begin by considering the "small power set" discussed earlier, that is, the set of all finite subsets of $A$, where $A$ is a polyhedral set. We denote the small power set of $A$ by $2^{A} .2^{A}$ is not itself a polyhedral set, but it can be realized as a formal union of disjoint polyhedral sets in spaces of increasing dimensionality. For instance, in the case where $A$ is an open (not necessarily bounded) interval, we can view $2^{A}$ as consisting of a point (the empty subset of $A$ ), an open interval (the set of singleton subsets of $A$ ), an open triangle (the set of 2 element subsets of $A$ ), an open 3-simplex (the set of 3-element subsets of $A)$, and so on. These constituent pieces have Euler measure $1,-1,1,-1, \ldots$ respectively. The terms do not yield a summable sequence, but there is no doubt what the incorrigible formalist Euler would have done: the series $1-1+1-1+\ldots$ is after all a geometric series, so it can be assigned a notional sum $1 /(1-(-1))=1 / 2$. More precisely, and more generally, given a polyhedral set $A$ one can define a formal power series $E(t)$ in which the coefficient of $t^{k}$ is the Euler measure of the set of $k$-element subsets of $A$. 
That is, $E(t)=\left(\begin{array}{c}m \\ 0\end{array}\right)+\left(\begin{array}{c}m \\ 1\end{array}\right) t+\left(\begin{array}{c}m \\ 2\end{array}\right) t^{2}+\ldots$ where $m=\chi(A)$. This is just the binomial expansion of $(1+t)^{m}$; when the integer $m$ is non-negative, the series is a polynomial whose value at $t=1$ is $2^{m}$, while for $m<0$ the series converges in an open disk of radius 1 to a function that extends to the whole complex plane away from $t=-1$, and whose value at $t=1$ is $2^{m}$. Either way, the value that one gets for $\chi\left(2^{A}\right)$ is $2^{\chi(A)}$. This "functoriality" is a sign that we are on the right track. (Here and elsewhere in the article, functoriality is said to hold when operations in the polyhedral category carry over, under the mapping $\chi$, to operations in the category of natural numbers, as described by Schanuel [Sch].)

Some less trivial corroboration of the validity of our approach comes from looking at $\left(\begin{array}{l}B \\ 2\end{array}\right)$, where $B$ is the power set of an open interval $A$. That is, we are looking at all the ways of choosing two distinct finite subsets $S_{1}, S_{2}$ of the interval $A$. Given $\sigma=\left\{S_{1}, S_{2}\right\} \in\left(\begin{array}{l}B \\ 2\end{array}\right)$, so that $S_{1} \neq S_{2}$, define the support-set (or support) of $\sigma$ as $S_{1} \cup S_{2}$, and define the rank of $\sigma$ as $\left|S_{1} \cup S_{2}\right|$. We define an Euler series $E(t)$ in which the coefficient of $t^{k}$ is the Euler measure of the rank- $k$ part of $\left(\begin{array}{c}B \\ 2\end{array}\right)$. Given a $k$-element subset $S$ of $A$, there are $3^{k-1}+3^{k-2}+\ldots+3+1$ ways to define $\sigma \in\left(\begin{array}{c}B \\ 2\end{array}\right)$ with support $S$. (Namely: for each $i$ between 0 and $k-1$ inclusive, one can assign the first $i$ elements of $S$ to both $S_{1}$ and $S_{2}$, the next element of $S$ to $S_{1}$ but not $S_{2}$, and each of the next $k-i-1$ elements of $S$ to $S_{1}$ only, $S_{2}$ only, or both $S_{1}$ and $S_{2}$, as one wishes. All $3^{k-1}+3^{k-2}+\ldots+3+1$ such pairs $S_{1}, S_{2}$ are distinct from one another. On the other hand, every pair of distinct finite subsets of $B$ whose union has cardinality $n$ can be written in this form in a unique way, by labelling the two sets as $S_{1}$ and $S_{2}$ so that the smallest element in their symmetric difference belongs to $S_{1}$.) Thus the Euler measure of the rank- $k$ part of $2^{A}$ is $\chi\left(\left(\begin{array}{l}A \\ k\end{array}\right)\right) \cdot\left(3^{k-1}+3^{k-2}+\ldots+3+1\right)$. The resulting Euler series $-t+(3+1) t^{2}-(9+3+1) t^{3}+\ldots$ analytically extends to the rational function $-t /(1+t)(1+3 t)$, whose value at $t=1$ is $-1 / 8=\left(\begin{array}{c}1 / 2 \\ 2\end{array}\right)$.

Let us agree to limit ourselves to taking power sets of subsets of $\mathbf{R}^{2}=\mathbf{R}$. (Note that the Euler measure of a polyhedral subset of $\mathbf{R}$ can be any integer, so not much generality has been lost through this restriction, in terms of the sort of numbers that will arise as Euler measures of derived sets.) We are 
going to consider sets of the form

$$
\begin{gathered}
\left(\begin{array}{c}
B \\
k_{1}
\end{array}\right), \\
\left(\begin{array}{c}
B \\
k_{1} \\
k_{2}
\end{array}\right) \\
\left(\begin{array}{c}
\left(\begin{array}{c}
B \\
k_{1} \\
k_{2}
\end{array}\right) \\
k_{3}
\end{array}\right),
\end{gathered}
$$

etc., where $A$ is a polyhedral subset of $\mathbf{R}$ and $B=2^{A}$; we will define them iteratively. We will call these sets gizmos (a more respectable term might be "selection schemes"). At each step, we will introduce a linear ordering on the gizmo, along with a support-set operation; together these allow us to proceed to the next step. Our notation for such gizmos will be $G\left(2^{A} ; k_{1}\right)$, $G\left(2^{A} ; k_{1}, k_{2}\right), G\left(2^{A} ; k_{1}, k_{2}, k_{3}\right)$, etc.

We start with $2^{A}$ itself. Given subsets $S, T$ of $A$ with $|S|=|T|$, we say that $S$ is less than $T$ if the smallest element in their symmetric difference belongs to $S$. (Equivalently: Writing $S=\left\{s_{1}, s_{2}, \ldots, s_{k}\right\}$ and $T=\left\{t_{1}, t_{2}, \ldots, t_{k}\right\}$ with $s_{1}<s_{2}<\ldots<s_{k}$ and $t_{1}<t_{2}<\ldots<t_{k}, S$ is less than $T$ if $s_{i}<t_{i}$ for the first location $i$ at which the two sequences differ.) The support-set operation from $2^{A}$ to itself is just the identity operation. Given a gizmo $G$ with a linear ordering and a support-set operation, and given an integer $k \geq 0$, we define the gizmo $\left(\begin{array}{c}G \\ k\end{array}\right)$ as the set of all sequences $\left(g_{1}, g_{2}, \ldots, g_{k}\right) \in G^{k}$ with $g_{1}<g_{2}<\ldots<g_{k}$ (with respect to the linear ordering on $G$ ). We order $\left(\begin{array}{l}G \\ k\end{array}\right)$ lexicographically (so that $\left(g_{1}, g_{2}, \ldots, g_{k}\right)$ is less than $\left(g_{1}^{\prime}, g_{2}^{\prime}, \ldots, g_{k}^{\prime}\right)$ if and only if $g_{i}<g_{i}^{\prime}$ at the first location $i$ at which the two sequences differ) and we define the support of $\left(g_{1}, \ldots, g_{k}\right)$ as the union of the supports of $g_{1}$ through $g_{k}$.

For any gizmo $G$, the number of elements of $G$ whose support is a particular $k$-element subset of $A$ does not depend on the particular subset of $A$, but is some number $n_{k}$ that depends only on $k$ (and $G$ ). We therefore define the Euler series of $G$ as

$$
\sum_{k=0}^{\infty} \chi\left(\left(\begin{array}{l}
A \\
k
\end{array}\right)\right) n_{k} t^{k} .
$$




\section{Continued Binomial Coefficients}

Theorem 1: The Euler series for $G\left(2^{A} ; k_{1}, k_{2}, \ldots, k_{r}\right)$ converges in a neighborhood of $t=0$ to a rational function of $t$ whose value at $t=1$ is the iterated binomial coefficient $C\left(2^{\chi(A)} ; k_{1}, k_{2}, \ldots, k_{r}\right)$ (defined by the initial condition $C(n)=n$ and the recurrence relation $C\left(n ; k_{1}, k_{2}, \ldots, k_{i}\right)=$ $\left.\left(\begin{array}{c}C\left(n ; k_{1}, k_{2}, \ldots, k_{i-1}\right) \\ k_{i}\end{array}\right)\right)$.

REMARK: While this result is not as general as one might like, it does back up my contention that my definition of the power set, or something like it, can interact in a functorially appropriate way with other more basic polyhedral operations, once the category of polyhedral sets and polyhedral maps has been extended in a suitable way.

Proof: As in the argument given at the end of Section 2, the proof of the main theorem uses inclusion-exclusion. We illustrate the proof for the concrete case $G\left(2^{\chi(A)} ; 2,2\right)$ where $A$ varies over the polyhedral subsets of $\mathbf{R}$, since it contains all the key ideas. An element of this gizmo can be described as a 4 -tuple $\left(S_{1}, S_{2}, T_{1}, T_{2}\right)$ of subsets of $A$ satisfying the following three conditions: (1) $S_{1}<S_{2}$; (2) $T_{1}<T_{2}$; (3) $\left\{S_{1}, S_{2}\right\}<\left\{T_{1}, T_{2}\right\}$. Note that condition (3) is equivalent to the condition that either (i) $S_{1}<T_{1}$ or else (ii) $S_{1}=T_{1}$ and $S_{2}<T_{2}$. We know that, in the case where $A$ is a finite set of points, the gizmo is itself just a finite set of points, and that its Euler series is a polynomial in $t$ whose value at $t=1$ is just $C\left(2^{\chi(A)} ; 2,2\right)$. Our goal is to show that this same formula applies even when $A$ is not a finite set of points, essentially by showing that the two cases are governed by the same inclusion-exclusion calculation.

Before we do the comparison, it is helpful to switch to a re-statement of the problem more amenable to inclusion-exclusion analysis. We will the

consider the "symmetrized gizmo" consisting of all 4-tuples obtained from the elements of $G\left(2^{\chi(a)} ; 2,2\right)$ by the action of the 8-element group that allows one to switch $S_{1}$ with $S_{2}, T_{1}$ with $T_{2}$, or the pair $S_{1}, S_{2}$ with the pair $T_{1}, T_{2}$. Note that the elements of the symmetrized gizmo are exactly the 4-tuples $\left(S_{1}, S_{2}, T_{1}, T_{2}\right)$ of subsets of $A$ for which none of the following conditions holds: (a) $S_{1}=S_{2}$; (b) $T_{1}=T_{2}$; (c) $S_{1}=T_{1}$ and $S_{2}=T_{2}$; (d) $S_{1}=T_{2}$ and $S_{2}=T_{1}$. Regardless of the nature of $A$ (finite vs. infinite), it is clear that the Euler series of the new gizmo is 8 times the Euler series of the old, so it is enough to prove that the Euler series of the symmetrized gizmo is a rational function of $t$ whose value at $t=1$ is given by a polynomial in $2^{\chi(A)}$. Once 
we have shown that the value at $t=1$ depends only on $\chi(A)$, and not on $A$, our observations for the case where $A$ is finite will complete the proof.

To prove the required properties of the Euler series for symmetrized gizmos, the principle of inclusion-exclusion guarantees that it is enough to show that the claim holds when we look at the set of 4-tuples in which some specified (possibly empty) subset of the forbidden conditions (a),(b),(c),(d) does in fact hold. But the effect of imposing any or all of these four conditions is merely to require that one is not choosing four subsets of $A$ but three, two, or one. Specifically, if we let $E_{a}$ denote the Euler series for the set of 4-tuples of (finite) subsets of $A$ for which condition (a) holds, etc., and we let $E(k)$ denote the Euler series for the set of $k$-tuples of subsets of $S$, then the Euler series for $G\left(2^{A} ; 2,2\right)$ is equal to $[E]-\left[E_{a}+E_{b}+E_{c}+E_{d}\right]+\left[E_{a b}+E_{a c}+E_{a d}+\right.$ $\left.E_{b c}+E_{b d}+E_{c d}\right]-\left[E_{a b c}+E_{a b d}+E_{a c d}+E_{b c d}\right]+\left[E_{a b c d}\right]=\left[E_{4}\right]-\left[E_{3}+E_{3}+E_{2}+E_{2}\right]+$ $\left[E_{2}+E_{1}+E_{1}+E_{1}+E_{1}+E_{1}\right]-\left[E_{1}+E_{1}+E_{1}+E_{1}\right]+\left[E_{1}\right]=E_{4}-2 E_{3}-E_{2}+2 E_{1}$. (Note that if we replace $E_{k}$ by $x^{k}$, the preceding expression reduces to

$$
8\left(\begin{array}{c}
x \\
2 \\
2
\end{array}\right)
$$

as it ought to.) Here we see that the Möbius coefficients that enter into the inclusion-exclusion formula are independent of the set $A$, but depend only on $k_{1}=2, k_{2}=2$. This independence is true more generally. Hence the entire problem reduces to the case of $\left(2^{A}\right)^{j}$, with $j$ varying; that is, the gizmo consisting of all ways of choosing a $j$-tuple of (not necessarily distinct) subsets $S_{1}, S_{2}, \ldots, S_{j}$ of $A$.

We can now conclude the proof. When $A$ is a finite set of points, the Euler series for $\left(2^{A}\right)^{j}$ is $\left(1+\left(2^{j}-1\right) t\right)^{\chi(A)}$, since for each of the $\chi(A)$ points of $A$ we can either choose to include it in none of the sets $S_{1}, S_{2}, \ldots, S_{j}$ or else choose to include it in some of the $j$ sets (which we can do in any of $2^{j}-1$ ways). When $A$ is an open interval, the Euler series for $\left(2^{A}\right)^{j}$ is $\left(1+\left(2^{j}-1\right) t\right)^{-1}=1-\left(2^{j}-1\right) t+\left(2^{j}-1\right)^{2} t^{2}-\ldots$, since for each subset of $A$ of size $i$, the number of ways of assigning each element of the subset to at least one (and possibly all) of the $j$ sets $S_{1}, S_{2}, \ldots, S_{j}$ is $\left(2^{j}-1\right)^{i}$. In general, the Euler series for $\left(2^{A}\right)^{j}$ is $\left(1+\left(2^{j}-1\right) t\right)^{\chi(A)}$, which when evaluated at $t=1$ gives $\left(2^{j}\right)^{\chi(A)}=\left(2^{\chi(A)}\right)^{j}$. Note that this is a polynomial in $2^{\chi(A)}$, as claimed. Hence all the gizmos in question (the original gizmo $G\left(2^{A} ; k_{1}, k_{2}, \ldots, k_{r}\right)$ and its symmetrized version) have Euler measure given by polynomials in $\chi(A)$, which we can identify by looking at the case where $A$ is a finite set. 
Theorem 1 in its current form is a bit eccentric in its focus. Ideally one would like to replace it by a result that says that the operation selecting a subset of size $k$ may be freely combined with the operations of disjoint union and Cartesian product (and perhaps other natural polyhedral operations as well) in such a fashion that all the resulting polyhedral sets satisfy formulas

like $\left(\begin{array}{c}\chi(A \\ k)=\left(\begin{array}{c}(A) \\ k\end{array}\right)\end{array}\right), \chi(A \cup B)=\chi(A)+\chi(B)-\chi(A \cap B)$, and $\chi(A \times B)=$ $\chi(A) \chi(B)$.

\section{Exponentiation}

If $A, B$ are polyhedral sets $\left(A \subset \mathbf{R}^{m}, B \subset \mathbf{R}^{n}\right)$, the set of affine maps from $A$ to $B$ has a well-defined Euler measure that can be associated with it in a number of ways. One simple way of defining it is to let $v_{1}, v_{2}, \ldots, v_{k}$ be points in $\mathbf{R}^{m}$ whose affine span contains $A$; then every map from $A$ to $B$ is determined by its values on $v_{1}, v_{2}, \ldots, v_{k}$, so that the set of affine maps from $A$ to $B$ becomes associated with a subset of $B^{k}$ (namely, the set of all $k$-tuples $\left(f\left(v_{1}\right), f\left(v_{2}\right), \ldots, f\left(v_{k}\right)\right)$ arising from affine maps $\left.f: A \rightarrow B\right)$. One can show that this set in $B^{k}$ is itself polyhedral, and that its Euler measure does not depend on the spanning set $v_{1}, v_{2}, \ldots, v_{k}$ that was chosen.

For example, in the case $A=(0,1) \subset \mathbf{R}$ it is natural to take $v_{1}=0$ and $v_{2}=1$. Assume that $B$ is compact. Then the set of affine maps from $A$ to $B$ corresponds to the set of pairs $\left(w, w^{\prime}\right) \in \mathbf{R}^{n} \times \mathbf{R}^{n}$ such that $t w+(1-t) w^{\prime} \in B$ for all $0<t<1$. It is not hard to show that this set is polyhedral and that for each $w \in \mathbf{R}^{n}$, the set $\left\{w^{\prime} \in \mathbf{R}^{n}: t w+(1-t) w^{\prime} \in B\right.$ for all $\left.0<t<1\right\}$ has Euler measure 1 or 0 according to whether or not $w \in B$ (this is where compactness gets used, via the fact that a starlike compact polyhedral set has Euler measure 1). Hence, by Fubini's Theorem, the set of affine maps from $(0,1)$ to $B$ has Euler measure $\chi(B)$.

Let us now take $A$ to be a polyhedral subset of $\mathbf{R}$ with no isolated points. (It is clear that the case of general polyhedral sets $A$ can be handled by combining the case in which $A$ is finite and the case in which $A$ has no isolated points.) Then any polyhedral map $f: A \rightarrow B$ is locally affine at all but a finite number of points. We call a point at which $f$ fails to be locally affine (whether by virtue of discontinuity or by virtue of non-differentiability) a breakpoint of $f$, and we call the number of breakpoints the rank of $f$. (Note that this notion of rank, specialized to the case $B=\{0,1\}$, coincides with the notion introduced in section 3: since we are assuming that $A$ has no isolated 
points, the indicator function of a finite subset of $A$ with cardinality $n$ has exactly $n$ points of discontinuity, namely, the elements of $A$ themselves.)

Let $S$ be a finite subset of $A$. We wish to count (in the sense of Eulermeasure) the polyhedral maps from $A$ to $B$ whose breakpoints are precisely the points of $S$. It is not hard to see that the set of maps from $A$ to $B$ that are locally affine on $A \backslash S$ is amenable to the same analysis as we applied in the first paragraph of this section; that is, it has a well-defined Euler measure. If a set is locally affine on $A \backslash S$, its breakpoints form a subset of $S$, and vice versa. Therefore the method of inclusion-exclusion gives us a way to calculate the Euler measure for the set of polyhedral maps from $A$ to $B$ with breakpoint-set $S$. It is easy to prove that this Euler measure has the same value for all $S$ of fixed size $k$. Call this value $n_{k}$. Then one can define the Euler measure of the set of polyhedral maps with exactly $k$ breakpoints as $\chi\left(\left(\begin{array}{l}A \\ k\end{array}\right)\right) \cdot n_{k}=\left(\begin{array}{c}\chi(A) \\ k\end{array}\right) n_{k}$, and define $E(t)=\sum_{k=0}^{\infty}\left(\begin{array}{c}\chi(A) \\ k\end{array}\right) n_{k} t^{k}$.

For example, suppose $A=(0,1), B=\{0,1\}$. A polyhedral map from $A$ to $B$ will have some number of breakpoints, say at $0<x_{1}<x_{2}<\ldots<x_{k}$ $(k \geq 0)$; in between these breakpoints, the map will be constant, since $B$ is discrete. I claim that the number of polyhedral maps from $A$ to $B$ with some fixed set of $k$ breakpoints is $2 \cdot 3^{k}$. For, starting at the left, the map can take either the value 0 or the value 1 on the interval $\left(0, x_{1}\right)$. Thereafter, for each $1 \leq i \leq k$, we can choose either value ( 0 or 1 ) for the function at $x_{i}$ and either value for the function on $\left(x_{i}, x_{i+1}\right)$, as long as we do not have both values equal to the value on $\left(x_{i-1}, x_{i}\right)$ (for then there would not be a breakpoint at $x_{i}$ ); there are always 3 ways to implement this. Hence the Euler series is $2-6 t+18 t^{2}-54 t^{3}+\ldots=2 /(1+3 t)$, whose value at $t=1$ is $2 / 4=2^{-1}=\chi(B)^{\chi(A)}$.

Continuing this example in the spirit of Theorem 1, we can look at the set of pairs of distinct maps from $(0,1)$ to $\{0,1\}$, where the rank of such a pair is the cardinality of the union of the sets of breakpoints of the two maps. Then one can check that the set of maps with $k$ specified breakpoints has Euler measure $2\left(2^{4}-1\right)^{k}-\left(2^{2}-1\right)^{k}=2 \cdot 15^{k}-3^{k}$, so that $E(t)=\frac{2}{1+15 t}-\frac{1}{1+3 t}$ and $E(1)=\frac{2}{16}-\frac{1}{4}=-\frac{1}{8}=\left(\begin{array}{c}1 / 2 \\ 2\end{array}\right)$ as expected.

Let us now focus on the case where $A=(0,1)$ and $B$ is a bounded polyhedral set. There are two sorts of exponentiation we will consider: the "Morelli exponential" (the set of all hedral maps from $A$ to $B$ ) and the "Schanuel exponential" (the set of all polyhedral maps from $A$ to $B$ ); for 
definitions, see section 1 . The two cases are similar, for in both cases, the set of affine maps from any sub-interval $\left(x_{i}, x_{i+1}\right)$ to $B$ has Euler measure $\chi(B)$. (In the Morelli case, this is trivial, since one is merely looking at constant maps; in the Schanuel case, one appeals to the analysis given in the second paragraph of this section.) This allows us to prove:

Theorem 2: Let $B$ be any bounded polyhedral set, and let $\mathcal{F}$ be the set of hedral maps from $(0,1)$ to $B$ or the set of polyhedral maps from $(0,1)$ to $B$. In either case, the regularized Euler measure of $\mathcal{F}$ is 0 if $\chi(B)=0$ and $1 / \chi(B)=\chi(B)^{-1}$ otherwise.

Proof: For every set $S$ of $k$ points in $(0,1)$, the set of hedral or polyhedral maps from $(0,1)$ to $B$ whose breakpoints lie in $S$ has Euler measure $\chi(B)^{2 k+1}$, with $k$ factors of $\chi(B)$ coming from the points in $S$ and $k+1$ factors coming from the intervals in between (by virtue of the fact mentioned in the paragraph preceding the statement of Theorem 2). Inclusion-exclusion on the lattice of subsets of $S$ implies that the set of maps from $(0,1)$ to $B$ whose breakpoint-set is $S$ has Euler measure $\chi(B)\left(\chi(B)^{2}-1\right)^{k}$. Hence $E(t)=\chi(B) /\left(1+\left(\chi(B)^{2}-1\right) t\right)$. If $\chi(B)=0$, all coefficients vanish; if $\chi(B) \neq 0, E(1)=\chi(B) /\left(\chi(B)^{2}\right)=1 / \chi(B)$.

\section{Final thoughts}

It is worth pointing out here that full functoriality is too much to expect; for instance, if $A$ has generalized Euler measure 1/2 and $B$ has Euler measure -1 , then it is hard to believe that one could come up with a theory that would assign to $C=B^{A}$ a generalized Euler measure $(-1)^{\frac{1}{2}}= \pm i$, and still harder to believe such a theory would know what to make of $C^{C}$ (given that $i^{i}$ takes on infinitely many values). Yet numerous examples demonstrate that as long as one does not push things too far, functoriality does hold.

I do not claim that my proposed definition of regularized Euler measure is the "right" one; if the reader finds its formulation artificial, I must to some extent agree. However, I think that the way the theory-in-progress hangs together indicates that there is probably a better-motivated definition that agrees with mine for the class of examples I consider. A homological formulation would be especially useful, and I hope some readers will pursue this line of investigation. 


\section{References}

[Ch1] B. Chen, The Gram-Sommerville and Gauss-Bonnet theorems and combinatorial geometric measures for noncompact polyhedra, Advances in Mathematics 91 (1992), 269-291.

[Ch2] B. Chen, On the Euler measure of finite unions of convex sets, Discrete and Computational Geometry 10 (1993), 79-93.

[Ha1] H. Hadwiger, Eulers Characteristik und kombinatorische Geometrie, J. reine angew. Math. 194 (1955), 101-110.

[Ha2] H. Hadwiger, Erweiterter Polyedersatz und Euler-Shephardsche Additionstheoreme, Abhandlungen Mat. Seminar Hamburg 39 (1973), 120-129.

[KR] D. Klain and G.-C. Rota, Introduction to geometric probability. Cambridge University Press, 1997.

[Len] H. Lenz, Mengenalgebra und Eulersche Charakteristik, Abh. Math. Sem. Univ. Hamburg 34 (1970), 135-147.

[McM] P. McMullen, The polytope algebra, Advances in Mathematics $\mathbf{7 8}$ (1987), 76-130.

[McS] P. McMullen and R. Schneider, Valuations on convex bodies, Convexity and its Applications, P. Gruder and J. Wills, eds., Birkhäuser, Basel 1983, pp. 160-247.

[Mor] R. Morelli, A theory of polyhedra, Advances in Mathematics $\mathbf{9 7}$ (1993), 1-73.

[Pro] J. Propp, Euler measure as generalized cardinality; arXiv: math.CO/ 0203289.

[Sch] S. Schanuel, Negative sets have Euler characteristic and dimension, in: Lecture Note In Mathematics \#1488, Springer, 1991.

[Sta] R. Stanley, Enumerative combinatorics I, Wadsworth, 1986. 